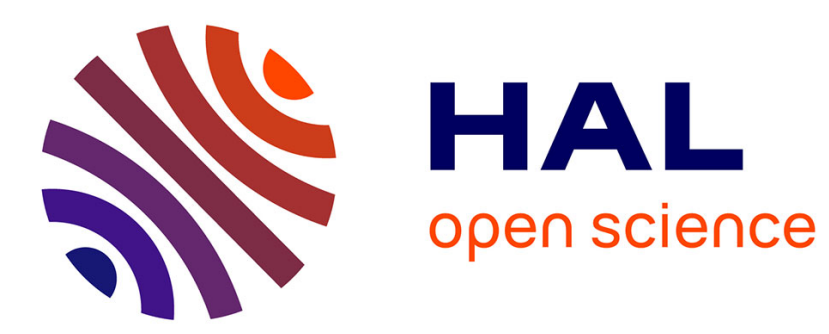

\title{
Texture-driven elastohydrodynamic bouncing
}

Thibault Chastel, Philippe Gondret, Anne Mongruel

\section{To cite this version:}

Thibault Chastel, Philippe Gondret, Anne Mongruel. Texture-driven elastohydrodynamic bouncing. Journal of Fluid Mechanics, 2016, 805, pp.577 - 590. 10.1017/jfm.2016.580 • hal-01398089

\section{HAL Id: hal-01398089 \\ https: / hal.sorbonne-universite.fr/hal-01398089}

Submitted on 16 Nov 2016

HAL is a multi-disciplinary open access archive for the deposit and dissemination of scientific research documents, whether they are published or not. The documents may come from teaching and research institutions in France or abroad, or from public or private research centers.
L'archive ouverte pluridisciplinaire HAL, est destinée au dépôt et à la diffusion de documents scientifiques de niveau recherche, publiés ou non, émanant des établissements d'enseignement et de recherche français ou étrangers, des laboratoires publics ou privés. 


\title{
Texture-driven elastohydrodynamic bouncing
}

\author{
Thibault Chastel ${ }^{1}$, Philippe Gondret ${ }^{2}$ and Anne Mongruel ${ }^{1} \dagger$ \\ ${ }^{1}$ Laboratoire de Physique et Mécanique des Milieux Hétérogènes (PMMH), UMR CNRS 7636 ; \\ PSL - ESPCI, 10 rue Vauquelin, 75005 Paris, France; Sorbonne Université - UPMC, Univ. \\ Paris 06; Sorbonne Paris Cité - UDD, Univ. Paris 07 \\ ${ }^{2}$ Laboratoire FAST, Univ. Paris-Sud, CNRS, Université Paris-Saclay, F-91405, Orsay, France
}

We investigate in detail the dynamics of bouncing of a fluid-immersed solid sphere onto a textured wall at moderate Reynolds and Stokes numbers. Using high-frequency interferometric measurements, the dynamics of the sphere is resolved in time and space, before, during and after collision with the wall. The critical Stokes number for bouncing is shown to be significantly influenced by the geometry of the texture, i.e. the surface fraction and the height of the micro-pillars. A modified Hertz model is developed to take into account the influence of this texture geometry on the collision dynamics. The predicted scaling for the collision time and penetration depth of the sphere into the textured wall is found to be in good agreement with the experimental measurements.

\section{Introduction}

The collision process of grains in a fluid is a key phenomenon for a good understanding of the complex dynamics of numerous industrial and natural multiphase flows, such as particle laden-flows, fluidised beds or submarine avalanches (Courrech du Pont et al. 2003). The immersed collision of grains is an elastohydrodynamic problem, first introduced by Davis et al. (1986) and later on considered by Lian et al. (1996) and Marshall (2011), which combines the fluid forces and the elastic deformation of the bodies. The theory in these articles predicted that bouncing can occur between smooth grains, even without physical contact, due to their elastic deformation from lubrication fluid pressure. In this problem, the dimensionless Stokes number, $S t$, defined as the ratio of grain inertia relative to fluid viscous forces, was expected to be a key parameter governing the collision process. Barnocky \& Davis (1988) first demonstrated experimentally, by dropping small spheres in air onto a wall covered by a thin layer of viscous liquid, that no bouncing is observed below a critical Stokes number, $S t_{c}$. Above this critical value, bouncing occurs and the coefficient of restitution of the collision, which is defined as the velocity modulus after the collision relative to its value prior to the collision, is non zero. With the use of high speed video cameras, Joseph et al. (2001) and Gondret et al. (2002) measured this coefficient of restitution in the case where the sphere in normal collision with the wall is fully immersed in a fluid. The coefficient of restitution was found to be an increasing function of the Stokes number above a critical value $S t_{c} \sim 10$. However, this critical value for bouncing and also the coefficient of restitution was shown by Joseph et al. (2001) to have non-negligible fluctuations generated by asperities of the solid surfaces. Thus, surface roughness has been considered by Yang \& Hunt (2008) using analytical

$\dagger$ Email address for correspondence: anne.mongruel@upmc.fr 

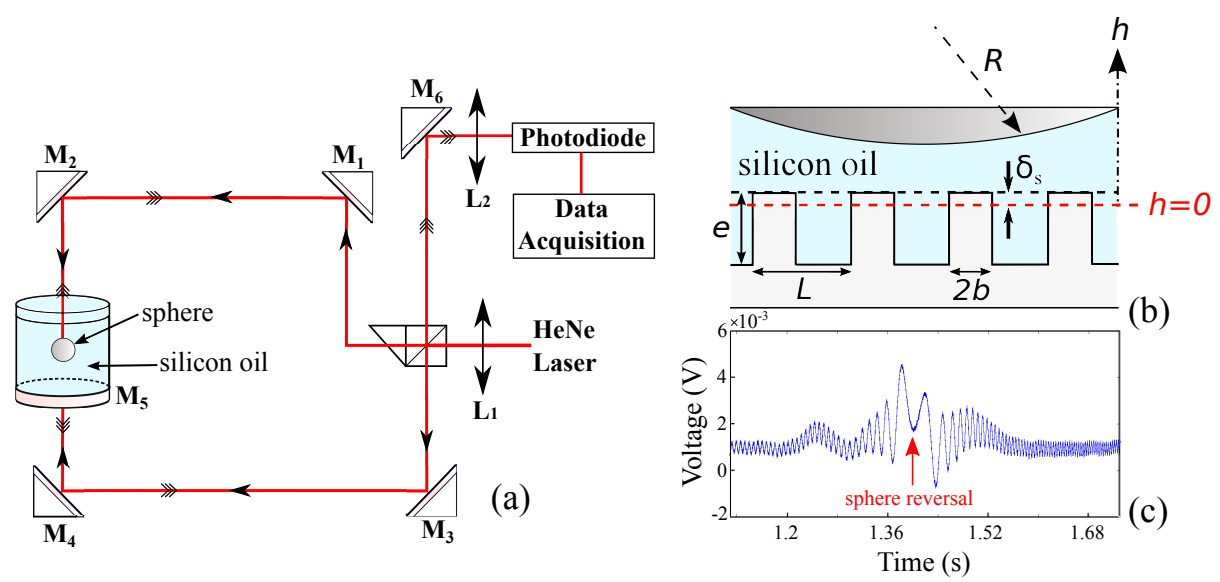

FiguRE 1. a) Sketch of the experimental set-up. b) Geometrical parameters involved in the sphere/textured wall collision. c) Example of interferometric signal in a bouncing case.

modeling, and in the numerical simulations by Ardekany \& Rangel (2008), Simeonov \& Calantoni (2012), Izard et al. (2014) and Costa et al. (2015).

The present work investigates how the collisional process of a solid sphere onto a wall in a viscous liquid is influenced by a wall texture, with a special attention given to the bouncing transition. The texture considered here consists of a network of square micropillars at the wall surface, whose geometrical parameters can be easily controlled and varied. To resolve the sphere motion not only before and after the collision but also during the collision, we use a high-frequency interferometric technique where the sphere acts as a moving mirror. Such an interferometric technique was already used by Mongruel et al. (2010) to investigate the near-wall dynamics of a sphere settling towards a smooth wall at finite Stokes and Reynolds numbers, just below the bouncing transition $\left(1<S t<S t_{c}\right)$. Recently, the influence of a wall texture on the near-wall dynamics of a sphere was studied with the same device for Reynolds and Stokes numbers ranging from very low (Chastel \& Mongruel 2016) to finite values (Chastel 2015), and it was found that the velocity of the sphere at a given distance from the top of the texture is larger than the corresponding value near a smooth wall. Here, we present new results for the collision dynamics of an immersed sphere onto a micro-textured wall around the bouncing transition. We first describe the experimental set-up and present the experimental data showing the dynamics of approach, of contact with the textured wall and of micro-rebounds. From these data, the time duration of the collision and the maximal penetration depth of the sphere into the wall texture can be measured, together with the impact and rebound velocities. A modified Hertz contact model is derived to take into account the geometry of the wall texture and then to predict the scaling laws for the collision time and penetration depth, which differ from the classical Hertz theory (see Johnson 1985). These predictions compare favourably with the experimental measurements.

\section{Experimental setup}

The experiments have been made with a high-frequency interferometric device, sketched in figure 1(a), originally designed by Lecoq et al. (1993). A solid sphere of density $\rho_{s}$ and radius $R$ settles under gravity in a silicon oil 47V1000 (from Bluestar silicons) of density $\rho=978 \mathrm{~kg} / \mathrm{m}^{3}$ and kinematic viscosity $\nu=10^{-3} \mathrm{~m}^{2} / \mathrm{s}$, at $25^{\circ} \mathrm{C}$, towards the horizontal bottom wall of a cylindrical container with a $50 \mathrm{~mm}$ diameter 
and a $40 \mathrm{~mm}$ height. This bottom wall can be either a smooth glass wall or a textured surface made of a thiolen resin (Norland Optical Adhesive NOA 81 of elastic modulus $E_{w} \simeq 1.4 \mathrm{GPa}$ ) obtained by photolithography and comprised of a forest of pillars with a height $e$ and a square base of side $2 b$ (figure 1b). These pillars are organised in a periodic square pattern of wavelength $L$, thus with the surface fraction $\phi=(2 b / L)^{2}$. Different textures are prepared by varying the geometrical parameters within the ranges $20 \leqslant e \leqslant 130 \mu \mathrm{m}, 50 \leqslant 2 b \leqslant 100 \mu \mathrm{m}$, and $140 \leqslant L \leqslant 240 \mu \mathrm{m}$, leading to an aspect ratio and surface fraction of the pillars in the range $0.1<e / 2 b<2.5$ and $0.05 \leqslant \phi \leqslant 0.3$ respectively. The sphere radius has been varied in the range $4 \leqslant R<8 \mathrm{~mm}$ so that the relative texture roughness lies in the range $10^{-3} \lesssim e / R \lesssim 10^{-2}$. The sphere material is either steel $\left(\rho_{s}=7.8 \times 10^{3} \mathrm{~kg} / \mathrm{m}^{3}, E_{s}=240 \mathrm{GPa}\right)$ or carbid tungsten $\left(\rho_{s}=15.6 \times 10^{3}\right.$ $\left.\mathrm{kg} / \mathrm{m}^{3}, E_{s}=550 \mathrm{GPa}\right)$ of roughness $\epsilon \simeq 0.1 \mu \mathrm{m}$ and $\epsilon \simeq 0.03 \mu \mathrm{m}$, respectively, which is negligible when compared to the pillar height $\left(\epsilon / e<10^{-3}\right)$ and also smaller than the light wavelength, leading to a mirror polish surface for the sphere.

The cylindrical container is filled with oil (no free surface) and inserted in an interferometric device, where the immersed sphere is used as a reflector (figure 1a). Before each experiment the sphere is held with a magnet at the center of the top plane window (made of glass of optical quality) of the fixed container. Removing this magnet initiates the sphere settling, and enables the pathway for the upper laser beam which reflects on the top of the settling sphere. This laser beam interfers with the other beam reflecting onto a plane mirror, that is fixed under the bottom wall of the container. As the sphere moves, the interference fringes move accordingly. The light intensity of the fringes is then converted into an electric signal by a photodiode, and recorded with an oscilloscope at a sampling frequency of $25 \mathrm{MHz}$. Detection of the successive maxima of this electric signal gives access to the sphere displacement $d h$ with a resolution of $\lambda / 2 n \simeq 0.23 \mu \mathrm{m}$, where $\lambda=0.633 \mu \mathrm{m}$ is the wavelength of the He-Ne laser beam, and $n=1.4$ is the optical index of the silicon oil. In practice, the maximum signal frequency that can be detected by the set-up is $0.5 \mathrm{MHz}$, yielding a time resolution of $0.002 \mathrm{~ms}$. The instantaneous sphere velocity $V(t)=-d h / d t$ can thus be measured in the range $10^{-4} \lesssim V \lesssim 10^{-1} \mathrm{~m} / \mathrm{s}$, with a relative error smaller than $1 \%$ in the approach phase and that does not exceed $5 \%$ in the collision and contact phase. The instantaneous position, $h(t)$, is obtained by integration, with the reference $h=0$ determined a posteriori as the final sphere rest position on the wall when $V=0$. It should be noted that when the sphere is resting on the surface, compressive stresses due to the apparent weight of the sphere deform the surface. For a glass wall, this deformation can be neglected. However, for a compliant material such as the NOA resin textures, we anticipate that the penetration of the sphere into the surface is non-negligible compared to the scale of observation. The static penetration depth of the sphere in the pillars is denoted $\delta_{s}$ (see figure $1 \mathrm{~b}$ ), and its value will be discussed in section (4.1). As the sphere slows down on approach to the wall, the recorded light modulation slows down accordingly until its complete arrest in the case of a "sticking" collision. In the bouncing case, the time modulation of the signal first displays a slowing down before an acceleration, which is the signature of the change of direction of the sphere (figure 1c).

In the present experiments, we investigate the elastohydrodynamic regime close to the bouncing transition in the range $2.5 \leqslant S t \leqslant 5.8$ (see Table 1 ), where the Stokes number is defined as $S t=(2 / 9) \rho_{s} R V_{T} / \eta$, with $\eta=\rho \nu$ the dynamic viscosity of the oil. Note that the present definition of $S t$ is based on $V_{T}$, the terminal velocity of the sphere in the corresponding unbounded fluid in contrast to other studies that used the velocity of the sphere just prior to impact. Here, $V_{T}$ can be inferred from the viscous "Stokes" drag force corrected from the small inertial effect known as Oseen's correction (see Mongruel 


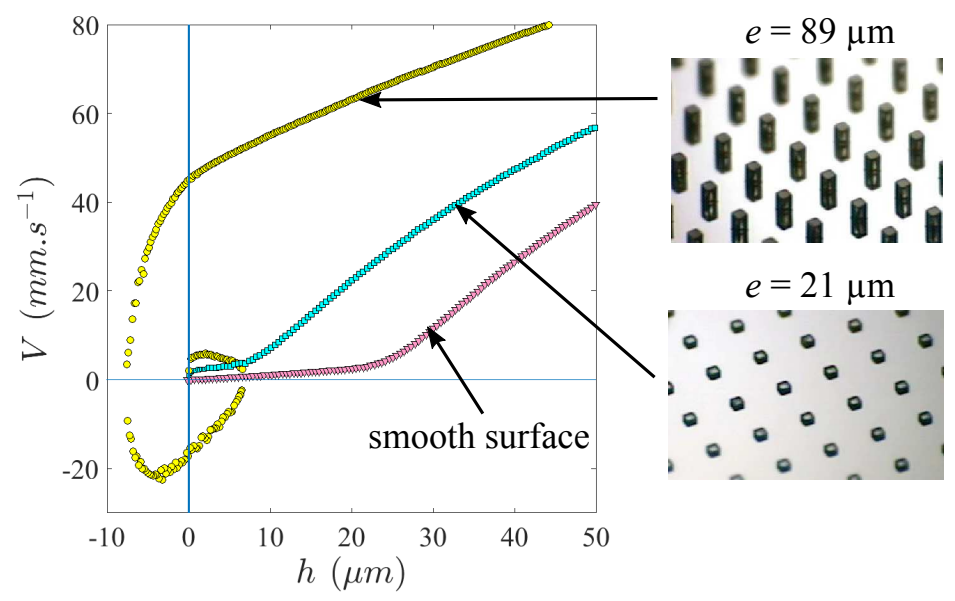

Figure 2. Velocity $V$ as a function of position $h$ (with $h=0$ for the sphere at rest on the wall) for a steel sphere $(2 R=14 \mathrm{~mm})$ settling in $47 \mathrm{~V} 1000$ silicon oil $\left(S t=4.7, R e=5.3, V_{T}=380\right.$ $\mathrm{mm} / \mathrm{s}$ ) towards an horizontal wall: smooth wall $(\nabla)$, textured wall with surface fraction $\phi=0.05$ and height of pillars $e=21 \mu m(\square)$ or $e=89 \mu m(\circ)$.

\begin{tabular}{ccccc}
\hline Material & $\begin{array}{c}2 R \\
(\mathrm{~mm})\end{array}$ & $\begin{array}{c}V_{T} \\
(\mathrm{~m} / \mathrm{s})\end{array}$ & Re & St \\
\hline stainless steel & 10.5 & 0.27 & 2.8 & 2.5 \\
& 12.7 & 0.34 & 4.3 & 3.8 \\
& 14 & 0.38 & 5.3 & 4.7 \\
& 15 & 0.40 & 6.0 & 5.4 \\
& 15.9 & 0.42 & 6.6 & 5.8 \\
carbid tungsten & 8 & 0.34 & 2.8 & 4.9 \\
\hline
\end{tabular}

TABLE 1. Parameters values for the different used spheres, of different materials and diameter $2 R$, settling in 47V1000 silicon oil. The Reynolds and Stokes numbers are based on the calculated terminal velocity $V_{T}$ of the sphere in the corresponding unbounded fluid.

et al. 2010). The corresponding Reynolds number $R e=(2 R) V_{T} / \nu=9\left(\rho / \rho_{s}\right) S t$ is indeed in the range $2.8 \leqslant R e \leqslant 6.6$ for the two density ratios used here $\left(\rho_{s} / \rho \simeq 8\right.$ and 16).

\section{Critical Stokes number for bouncing}

Figure 2 shows the evolution of the sphere velocity, $V$, as a function of the sphere position, $h$, for a given sphere settling towards three different wall textures at the same Stokes number $S t=4.7$. As stated before, the Stokes number is defined using the terminal velocity of the sphere, and hence is fixed by the physical properties of the sphere $\left(\rho_{s}, R\right)$ and of the oil $(\rho, \nu)$. Thus, at a given Stokes number, the dynamics of the sphere far from the wall, i.e. at a distance from the wall larger than one sphere radius, is the same for the three experiments. By contrast, the near-wall dynamics of the sphere is dramatically influenced by the type of texture. In the case of a smooth wall, $(e=0, \phi=$ 0 ), the sphere velocity drops towards zero for vanishing distance, with a linear terminal 
regime that results from the lubrication flow in between the smaller and smaller gap, eventually dissipating all of the kinetic energy of the sphere (see Mongruel et al. 2010). This observation is consistent with the Stokes number here being subcritical $(S t \lesssim 10)$. In the case of a textured wall $(e \neq 0, \phi \neq 0)$, the velocity of the sphere is significantly larger, for a given distance $h$, than the corresponding velocity for a smooth wall. This is a consequence of the fluid flow in between the pillars, which decreases the pressure in the gap and thus the resisting lubrication force on the sphere. The velocity in fig. 2 appears to be shifted along the horizontal axis, with a shift length that is proportional to the pillar height, $e$, but also depends on $\phi$ and $b / L$ (Chastel 2015). When the height of the textures is small enough (e.g., $e=21 \mu \mathrm{m}$ for $\phi=0.05$ in fig. 2), and despite this near-wall enhancement of velocity, the sphere velocity still drops towards zero at vanishing distance $h$, but with a new ultimate regime which corresponds now to the lubrication flow in between the sphere and the top surface of the closest pillar (see Chastel \& Mongruel 2016). Strikingly, when the height of the texture is large enough (e.g., $e=89 \mu \mathrm{m}$ for $\phi=0.05$ ), the local velocity enhancement of the sphere is such that the contact with the top of the pillars arises with a non-zero impact velocity $V_{i} \simeq 5$ $\mathrm{cm} / \mathrm{s}$. Note that this impact velocity is significantly smaller than the terminal velocity $V_{T} \simeq 38 \mathrm{~cm} / \mathrm{s}$ due to the lubrication forces acting during the wall approach. In that case of non zero-impact velocity, the sphere rebounds, whereas bouncing was precluded in the two previous cases. Indeed, negative $h$ values observed in figure 2 correspond to the dynamical extra compression of the pillars during the collision.

As the elastic modulus of the sphere is much larger than the elastic modulus of the textures, the elastic deformation of the sphere is negligible when compared to the elastic deformation of the textures. The non-zero kinetic energy of the sphere when touching the textured wall is stored by the elastic deformation of the textures. The dynamic deformation is maximum (here $h \simeq-0.01 \mathrm{~mm}$ ) when the sphere velocity vanishes. The stored elastic energy turns back into kinetic energy as the sphere velocity becomes increasingly negative and the sphere takes off the wall with a non-zero rebound velocity: $V_{r} \simeq-2 \mathrm{~cm} / \mathrm{s}$ at $h \simeq 0$. The impact and rebound velocity measurements lead to the restitution coefficient $\left|V_{r} / V_{i}\right| \simeq 0.4$, which is significantly smaller than one. The height of the micro-rebound of the sphere in the fluid is $h \simeq 8 \mu \mathrm{m}$. The sphere then returns to the wall with a vanishing velocity at contact so that no subsequent rebound is possible: the sphere comes to rest at the "attractor" point $(h=0, V=0)$. Note that the $V(h)$ curve in the bouncing case has the same spiral shape as predicted from the calculations of Lian et al. (1996).

Figure 2 shows that bouncing is observed when the impact velocity is non-zero. Hence, the bouncing of a given sphere in a given fluid (i.e. at a given Stokes number) can be triggered by the wall texture as a result of the local velocity enhancement, e.g., here, beyond a critical value of the pillar height $e$ for a given pillar fraction $\phi$. Moreover, for a given $e$, bouncing occurs at Stokes number values decreasing with decreasing $\phi$, as a result of the local velocity enhancement being more pronounced for smaller $\phi$ values. The different cases are summarized in figure 3 where the bouncing and non-bouncing ("sticking") domains are reported in the $(e / 2 b, S t)$ plane for three $\phi$ values ranging from 0.3 down to 0.05 . The critical Stokes number for bouncing, $S t_{c}$, is thus situated at the boundary between those two domains and appears as a dashed line in fig. 3 . The results show that $S t_{c}$ depends both on $e / 2 b$ and $\phi$ with measured values ranging typically from 3 to 5 for the texture geometries investigated here. These values are significantly smaller than the value $S t_{c} \simeq 10$ reported previously in the case of smooth surfaces (Joseph et al. 2001 and Gondret, Lance \& Petit 2002). This shows quantitatively that the wall texture influences the rebound of particles on a wall in a fluid. This influence is a direct 

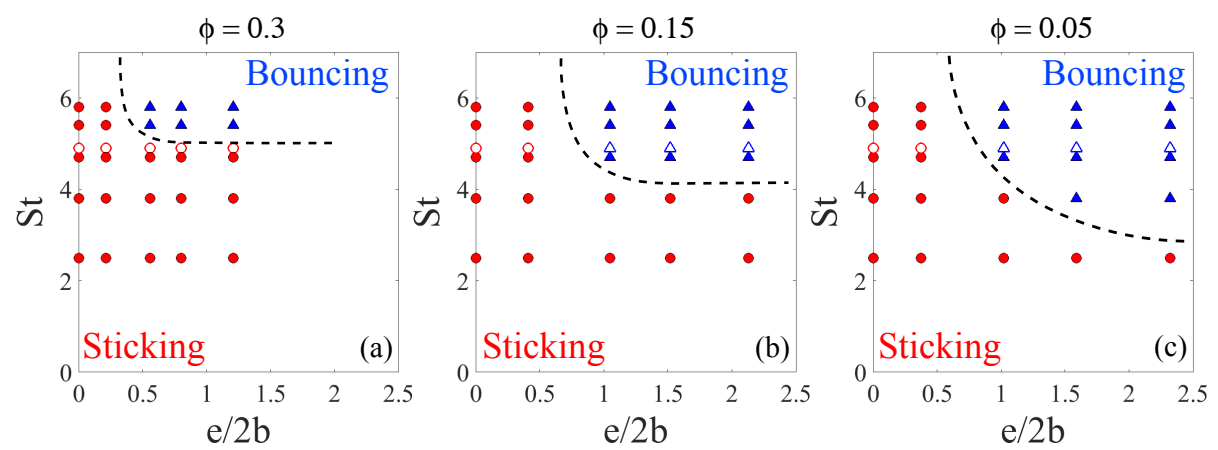

Figure 3. Bouncing $(\boldsymbol{\Lambda}, \triangle)$ or sticking $(\bullet, \circ)$ collision in the $(e / 2 b, S t)$ plane for the settling of a steel (filled symbols) or tungsten carbide (open symbols) sphere of different diameters and thus different Stokes numbers St in 47V1000 silicon oil towards a wall with micro-pillars of different aspect ratio $e / 2 b$ and different surface fraction (a) $\phi=0.30$, (b) $\phi=0.15$, (c) $\phi=0.05$. The dashed lines are only a guide for the eye.

consequence of the modification brought by the wall texture to the dynamics of approach of the sphere just prior to touching the wall.

Let us discuss two phenomena that may arise in the present sphere/wall collisions: non-Newtonian behavior of the oil and cavitation. Indeed, the high viscosity oil used here is known to be shear-thinning for high shear rates $\dot{\gamma}$ (Marston et al. 2010). It has a Newtonian behaviour with a constant viscosity for low enough shear rates $(\dot{\gamma} \lesssim 2500$ $\mathrm{s}^{-1}$ ) but exhibits a viscosity decrease beyond and, e.g., for $\dot{\gamma} \sim 10^{4} \mathrm{~s}^{-1}$, the viscosity value is about $85 \%$ of the low shear viscosity. The shear rate $\dot{\gamma}$ in the lubricated squeezed flow of thickness $h$ between the sphere and the wall can be estimated to be maximum at a distance $r_{0}=(2 R h)^{1 / 2}$ from the axis of the sphere, where the tangential flow velocity is equal, from mass conservation, to $V r_{0} / 2 h$. The highest value of the shear rate, $\dot{\gamma} \sim V r_{0} / 2 h^{2}$, can be estimated for the minimum gap thickness $h=e$ in the case of a textured wall, and $h=\epsilon$ in the case of a "smooth" wall. For typical values of sphere impact (e.g., $2 R=14 \mathrm{~mm}), \dot{\gamma} \sim 2400 \mathrm{~s}^{-1}$ for a typical textured wall $(e=100 \mu \mathrm{m}, V=$ $40 \mathrm{~mm} / \mathrm{s})$, and $\dot{\gamma} \sim 7000 \mathrm{~s}^{-1}$ for a typical "smooth" plane $(\epsilon=1 \mu \mathrm{m}, V=0.2 \mathrm{~mm} / \mathrm{s})$. We thus conclude that the variation of the oil viscosity during impact is negligible in our case. Concerning cavitation, this phenomenon was observed during the rebound of a sphere from a wetted surface by Marston et al. (2011). According to the criterion of Joseph (1998), cavitation is only expected when the tensile stress that can be estimated to be $2 \eta \dot{\gamma}$ exceeds an atmospheric pressure of approximately $10^{5} \mathrm{~Pa}$, which is not the case in our impact experiments, given the above values of $\dot{\gamma}$. Thus, the present experiments are not affected by non-Newtonian behavior or cavitation.

In the next section, we focus on the influence of the texture on the collision dynamics.

\section{Collision dynamics}

In the bouncing case of figure 2, the time evolution of the sphere velocity, $V(t)$, and of the sphere position, $h(t)$, are displayed in figure 4 . As already mentioned in section 2 , the sphere position is defined with a reference origin $h=0$ taken from the final position of the sphere at rest. The top position of the textures thus corresponds to a small positive value $h=\delta_{s}$ calculated from the model presented below. The maximal deformation of the texture $\delta_{\max }$ during the sphere/wall collision is measured in fig. $4 \mathrm{~b}$ from the minimum of the sphere position $h=\delta_{s}-\delta_{\max }$ when the sphere velocity 

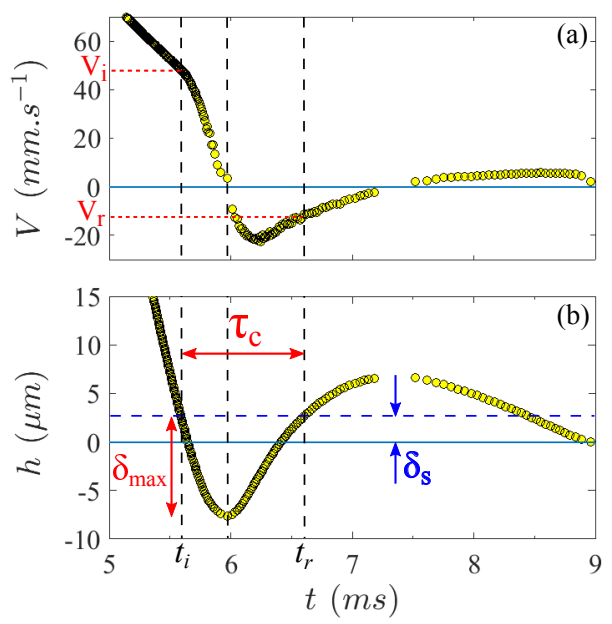

Figure 4. (a) Time evolution of the velocity $V(t)$ of a steel sphere $(2 R=14 \mathrm{~mm})$ settling in $47 \mathrm{~V} 1000$ silicon oil $\left(S t=4.7, R e=5.3, V_{T}=380 \mathrm{~mm} / \mathrm{s}\right)$ and colliding with a textured wall $(\phi=0.05, e=89 \mu \mathrm{m})$. (b) Corresponding time evolution of the sphere position $h(t)$ with $h=0$ for the sphere at final rest and top of the textures at $h=\delta_{s} \simeq 2.5 \mu \mathrm{m} . V_{i} \simeq 48 \mathrm{~mm} / \mathrm{s}, V_{r} \simeq-13$ $\mathrm{mm} / \mathrm{s}, \delta_{\max } \simeq 10 \mu \mathrm{m}, \tau_{c} \simeq 1 \mathrm{~ms}$.

vanishes (fig. 4a). The corresponding time appears as the vertical middle dashed line in fig. 4. The time $t_{i}$ (resp. $t_{r}$ ) at which the sphere touches (resp. takes off) the top of the texture at $h=\delta_{s}$ can be determined from fig. $4 \mathrm{~b}$ as the intersection of the $h(t)$ experimental curve with the estimated $h=\delta_{s}$ line. The collision duration $\tau_{c}$ is measured as $\tau_{c}=t_{r}-t_{i}$ and the impact and rebound velocity are measured from figure $4 \mathrm{a}$ as $V_{i}=V\left(t_{i}\right)$ and $V_{r}=V\left(t_{r}\right)$, respectively. In the typical bouncing of figure 4, the maximal sphere penetration is $\delta_{\text {max }} \simeq 10 \mu \mathrm{m}$, the collision duration is $\tau_{c} \simeq 1 \mathrm{~ms}$, the impact and rebound velocity are $V_{i} \simeq 48 \mathrm{~mm} / \mathrm{s}$ and $V_{r} \simeq-13 \mathrm{~mm} / \mathrm{s}$, leading to a restitution coefficient value of about 0.27 .

These plots enable the quantitative measurements of both the maximal penetration depth, $\delta_{\max }$, and the collision duration, $\tau_{c}$. The collision duration of a sphere with a wall has been already measured with a force transducer by Falcon et al. (1998) in the dry case and by Legendre et al. (2006) in the fully immersed case, but this technique did not give access to the penetration depth. Falcon et al. (1998) also discussed in details the influence of gravity during the collision and presented a gravity-modified Hertz interaction law. Before presenting our measured values of $\delta_{\max }$ and $\tau_{c}$ with varying $\rho_{s}, R, V, e$ and $\phi$, we first describe our modeling of the static and dynamic penetration of a sphere into a textured wall taking into account the gravity effect.

\subsection{Modeling the collision of the sphere with a textured wall}

Given the materials used here for the sphere and the textured wall $\left(E_{w} \ll E_{s}\right)$, we will consider in the modeling that only the wall is deformable whereas the sphere can be considered as perfectly rigid. The model developed in the following is very close to the original one developed by Hertz for the force contact law and collision dynamics of a sphere of radius $R$ and mass $m=(4 / 3) \pi \rho_{s} R^{3}$ with a smooth wall (Johnson 1985). Let $\delta_{0}(t)$ be the penetration of the bottom apex of the sphere in the pillars (Figure 5 ). For a given penetration $\delta_{0}$, the sphere/wall contact exists within a disk area of radius $a \simeq$ $\left(2 R \delta_{0}\right)^{1 / 2}$ and the local sphere penetration is $\delta(r)=\delta_{0}\left[1-(r / a)^{2}\right]$ at the radial distance $r$ from the bottom apex of the sphere (Figure 5). By assuming that the deformation 


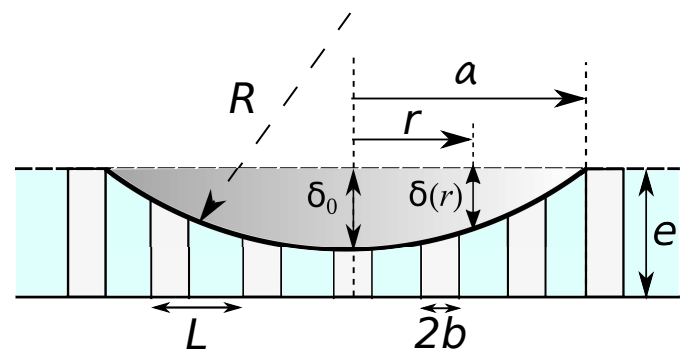

FIGURE 5. Sketch of the sphere penetrating into the pillars and definition of the geometrical parameters used in the modeling.

of the pillars remains in the elastic regime, the pillar deformation $\delta(r) / e$ generates the normal stress $\sigma(r)$ such as $\sigma(r)=E_{w} \delta(r) / e$. By taking into account the surface fraction of pillars, $\phi$, the total force exerted by the pillars onto the sphere is thus given by $F=\int_{0}^{a} \phi \sigma(r) 2 \pi r d r$, which leads to the normal force

$$
F=\pi \phi E_{w} \frac{R}{e} \delta_{0}^{2} .
$$

Note that this force contact law is non-linear with the sphere penetration $\delta_{0}$ due to the increasing contact area, but it differs from the non-linear Hertz scaling $F \sim \delta_{0}^{3 / 2}$. This force contact law can be first used in the case of a static contact, to calculate the penetration $\delta_{0}=\delta_{s}$ for a sphere at rest. In that case, the normal force $F$ exerted by the compressed pillars on the sphere balances the apparent gravity forces of the sphere $(4 / 3) \pi R^{3}\left(\rho_{s}-\rho\right) g$, which leads to

$$
\delta_{s}=R\left[\frac{4 e\left(\rho_{s}-\rho\right) g}{3 \phi E_{w}}\right]^{1 / 2} .
$$

Depending on the different spheres and the textures used in the present experiments, the static sphere penetration varies in the range $0.8 \leqslant \delta_{s} \leqslant 3 \mu \mathrm{m}$.

Next, we consider a settling sphere impacting a horizontal textured wall at a velocity $V_{i}$, as measured in the experiments (Figure 4). Before contacting the wall, the deceleration of the sphere is solely due to its hydrodynamic interaction with the wall, i.e. by the resistance of the lubricated interstitial fluid to be squeezed out of the gap between the sphere and the wall. During the contact, a fluid flow between the pillars still occurs, but for simplicity we assume here that the main contribution to the further deceleration of the sphere comes from the resistance of the pillars to elastic compression. We also neglect any other source of dissipation during the collision, such as vibrations. Under these assumptions, the conservation equation for the sphere energy can be written as

$$
\frac{1}{2} m V(t)^{2}+\frac{\pi}{3} \phi E_{w} \frac{R}{e} \delta_{0}(t)^{3}-\frac{4}{3} \pi R^{3}\left(\rho_{s}-\rho\right) g \delta_{0}(t)=\frac{1}{2} m V_{i}^{2} .
$$

In equation (4.3), the first term is the instantaneous kinetic energy of the sphere with the velocity $V(t)=d \delta / d t$, the second term is the instantaneous elastic energy corresponding to $\int_{0}^{\delta(t)} F d \delta$, and the third term is the instantaneous reduced gravitational energy. If we neglect any source of dissipation during collision, the sum of theses three term must be equal to the initial kinetic energy $(1 / 2) m V_{i}^{2}$ of the sphere when just touching the textures. The maximal penetration depth of the sphere is reached at the time at which the sphere velocity $V(t)$ is zero. At that time, the elastic energy is maximal and corresponds to the initial kinetic energy plus the additional gravitational energy. 
Let us first consider the case where the gravitational energy is zero or negligible compared to the initial kinetic energy. Hence, the corresponding value of the maximal penetration, $\delta_{m 0}$, reads as

$$
\delta_{m_{0}}=\left(\frac{2 \rho_{s} e R^{2} V_{i}^{2}}{\phi E_{w}}\right)^{1 / 3} .
$$

The corresponding time of collision, $\tau_{c_{0}}$, can be calculated from equation (4.3) without the gravity term, as $\tau_{c_{0}}=2 \tau \int_{0}^{1}\left(1-\tilde{t}^{3}\right)^{-1 / 2} d \tilde{t}$ where $\tilde{t}=t / \tau$ and $\tau=\delta_{m_{0}} / V_{i}$ is the leading order time scale. The integral term, denoted $I_{0}$, can be expressed with $\Gamma$ functions as $I_{0}=\pi^{1 / 2} \Gamma(4 / 3) / \Gamma(5 / 6) \simeq 1.4$ so that the time of collision can be written as

$$
\tau_{c_{0}} \simeq 2 I_{0}\left(\frac{2 \rho_{s} e R^{2}}{\phi E_{w} V_{i}}\right)^{1 / 3} .
$$

When the static penetration $\delta_{s}$ is of the same order of magnitude as $\delta_{m_{0}}$, the apparent weight of the sphere cannot be neglected during the collision. In that case, the maximal penetration depth of the sphere $\delta_{\max }$ is given from the complete equation (4.3) by

$$
\delta_{m_{0}}=\delta_{\max }\left[1-3\left(\frac{\delta_{s}}{\delta_{\max }}\right)^{2}\right]^{1 / 3} .
$$

For $\delta_{s}=0$ (no gravity), one recovers $\delta_{\max }=\delta_{m_{0}}$, whereas $\delta_{\max }>\delta_{m_{0}}$ for $\delta_{s} \neq 0$.

In the gravity case, the time of collision, $\tau_{c}$, can be calculated from complete equation (4.3) as $\tau_{c}=2 \tau \int_{0}^{1}\left[1-\tilde{t}^{3}+3\left(\delta_{s} / \delta_{m_{0}}\right)^{2} \tilde{t}\right]^{-1 / 2} d \tilde{t}$. The integral term, denoted $I_{g}$, can be calculated numerically for each value of the ratio $\delta_{s} / \delta_{m_{0}}$. Finally, the time of collision reads as

$$
\tau_{c} \simeq \tau_{c_{0}} \frac{I_{g}}{I_{0}} .
$$

Note that the above analysis is restricted to low enough impact velocities, i.e. $V_{i} \ll c^{*}$ where $c^{*}=\left(E_{w} / \rho_{s}\right)^{1 / 2}$ is a mixed characteristic velocity which does not correspond to the sound speed either in the sphere nor in the pillars. As $\left(E_{w} / \rho_{s}\right)^{1 / 2}>400 \mathrm{~m} / \mathrm{s}$ for the present experiments, this condition is largely fulfilled.

Note also that the predicted scalings given for the maximal penetration depth and time of collision without gravity effect by equations (4.4) and (4.5) are significantly different from the classical Hertz scalings $\delta_{m_{0}} \sim\left(\rho_{s} / E_{w}\right)^{5 / 2} R V^{4 / 5}$ and $\tau_{c_{0}} \sim\left(\rho_{s} / E_{w}\right)^{5 / 2} R V^{-1 / 5}$. The present model predicts in particular the scaling with the texture characteristics, i.e. the texture height $e$ and the surface density $\phi$, which are not embedded in Hertz theory. In addition, the influence of gravity on this scaling is described by equations (4.6) and (4.7).

\subsection{Comparison with experiments}

To test the prediction of the model relative to the collision duration, $\tau_{c}$, and penetration depth, $\delta_{\max }$, with the different parameters, we have done a large number of experiments with different spheres and different texture geometries, thus varying $\rho_{s}, R, e$, and $\phi$. Figure 6 shows four typical experiments with either a steel or tungsten carbide sphere colliding onto textures of different heights $e$ and different $\phi$. In each bouncing case, we observed the same characteristic spiral curve as in the bouncing case of figure 2 . These curves can been glimpsed from the experimental data of Marston et al. (2010) obtained with a high speed video camera but the present interferometric technique gives 

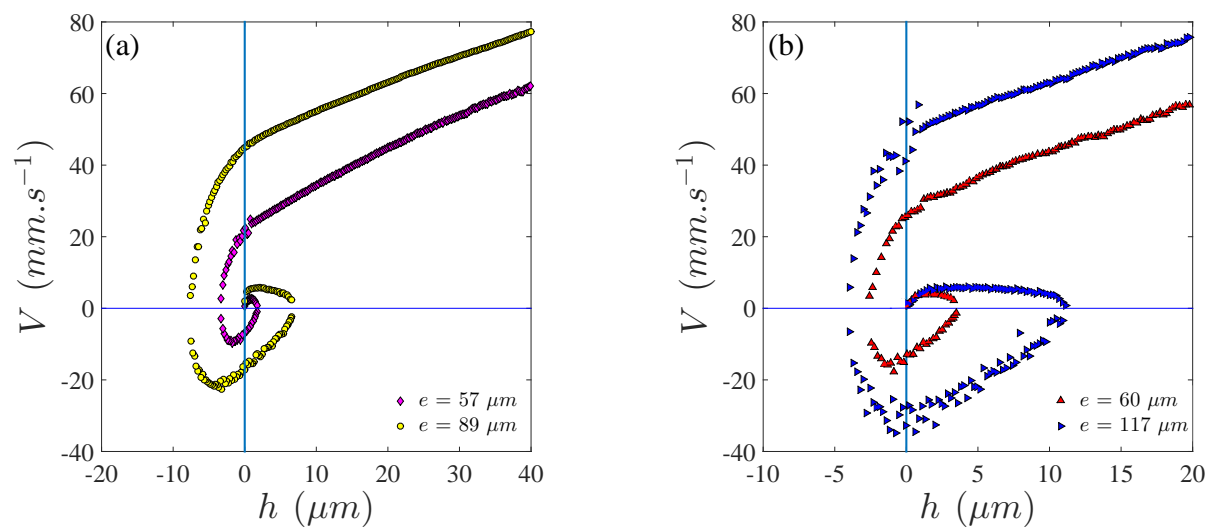

FiguRE 6 . Sphere velocity $V$ as a function of the sphere position $h$ (with $h=0$ for the sphere at final rest) for settling in $47 \mathrm{~V} 1000$ silicon oil towards textured walls with different surface fractions $\phi$ and heights $e$ of pillars. (a) Steel sphere $(2 R=14 \mathrm{~mm}$ and $S t=4.7)$ with $\phi=0.05$ and $e=57 \mu m(\checkmark)$ or $e=89 \mu m(\circ)(b)$ Tungsten carbide sphere $(2 R=8 \mathrm{~mm}$ and $S t=4.9)$, with $\phi=0.15$, and $e=60 \mu m(\Delta)$ or $e=117 \mu m(\bullet)$

much more detail during the collision, particularly in the $h \lesssim 0$ domain of compression. Note that in all the present experiments, the pillar deformation is smaller than about $10^{-1}$, thus remaining essentially in the elastic regime. By repeating several impacts on the same texture, we did not observe any significant changes in the resulting curves. This corroborates that the pillars remain in their elastic regime of deformation and that no significant plastic deformation occurs. In addition, considering the pillar aspect ratio, the dynamical contact force induced by the collision always remains smaller than the critical value, $F_{c}=\pi^{2} E_{w}(2 b)^{4} / 3 e^{2}$, given by the Euler criterion for the buckling instability (Landau \& Lifshitz 1986). Indeed, this criterion leads to the condition $\delta_{m_{0}} / e<$ $\left(\pi^{2} / 3\right)(2 b / e)^{2}$ which is less restrictive than the elastic condition mentioned above for the textures investigated here $(2 b / e>0.4)$. The order of magnitude of the viscous drag force on the sphere during the solid contact is negligible when compared to the order of magnitude of the elastic force. Indeed a rough estimate scaling $6 \pi \eta V_{i} R^{2} / e$ for this viscous force is smaller than the elastic force scaling $\pi \phi E_{w} \delta_{m_{0}}^{2} R / e$ at the condition $S t_{i}>$ $12 \eta^{2} /\left(\phi E_{w} \rho_{s} e^{2}\right)$, where $S t_{i}$ is here the Stokes number based on the impact velocity $V_{i}$. In the present experiments, this condition implies typically $S t_{i}>3.10^{-3}$ which is largely fulfilled considering the experimental $S t_{i}$ range $0.23 \lesssim S t_{i} \lesssim 1.3$. The assumptions, under which the above model has been derived, are thus properly met. The number of pillars implied in the solid contact is $N \simeq \pi a^{2} / L^{2}$, which varies in the range $2 \lesssim N \lesssim 30$ in the present experiments.

To test the scaling laws predicted by the model, we show in figure 7 the results of the maximal penetration depth and collision duration in the case of zero gravity. The experimental values $\delta_{m_{0}}$ and $\tau_{c_{0}}$ are calculated by equations (4.6) and (4.7) from the measured values $\delta_{\max }$ and $\tau_{c}$. When normalized with the length scale $\left(e R^{2} / \phi\right)^{1 / 3}, \delta_{m_{0}}$ is plotted in figure $7 \mathrm{a}$ as a function of the impact velocity $V_{i}$ normalized by the mixed "sound" velocity $c^{*}=\left(E_{w} / \rho_{s}\right)^{1 / 2}$. Note that, as the $\rho_{s}$ value for tungsten carbide is about twice of that for steel, the corresponding $c^{*}$ values do not differ much for the two materials. Hence, the experimental variations of the velocity ratio $V_{i} / c^{*}$ (approximately one decade) come from $V_{i}$ variations. The experimental error bars mainly arise from the determination of the final sphere position at rest $(h=0)$. In most cases, this 

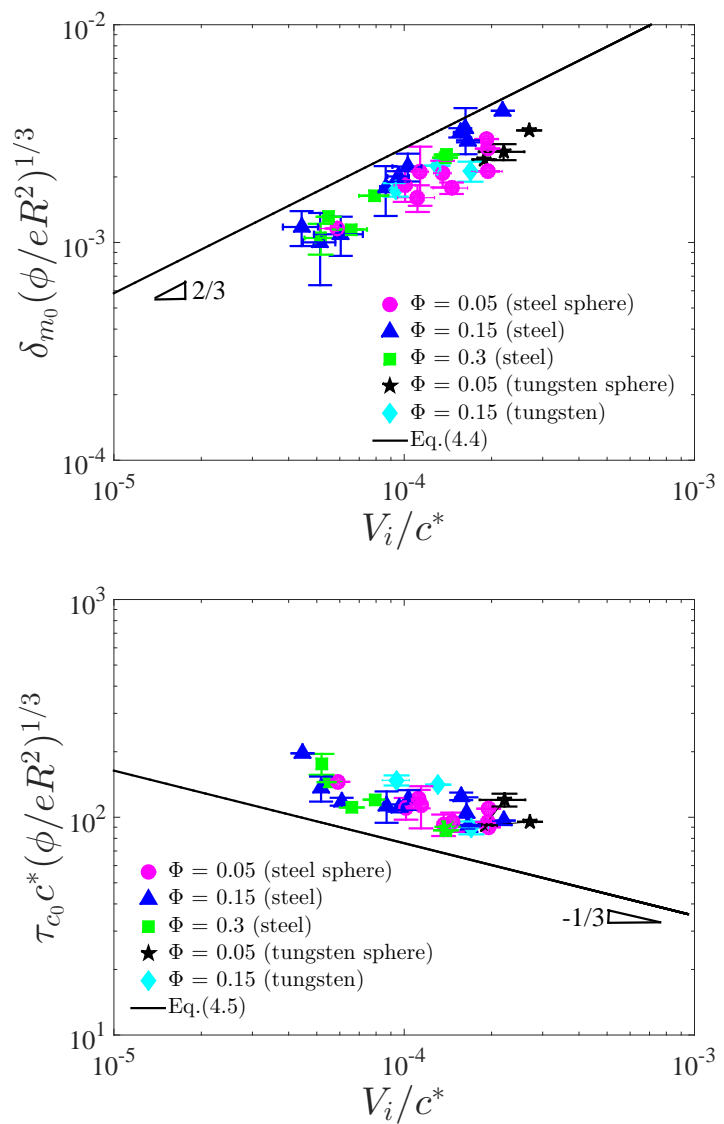

FiguRE 7. (a) Dimensionless maximal penetration depth $\delta_{m_{0}}\left(\phi / e R^{2}\right)^{1 / 3}$ as a function of the dimensionless impact sphere velocity $V_{i} / c^{*}$. Experimental values from $\delta_{\max }$ measurements and equation 4.5 for steel and tungsten carbid spheres onto different textures (data symbols) together with model equation $4.3(-)$. (b) Corresponding dimensionless collision duration $\tau_{c_{0}} c^{*}\left(\phi / e R^{2}\right)^{1 / 3}$. Experimental values from $\tau_{c}$ measurements and equation 4.6 (same data symbols) together with model equation $4.4(-)$.

determination is done with an error much smaller than $1 \mu \mathrm{m}$, leading to small error bars, except when some residual motion of the sphere is present when the error can reach up to about $3 \mu \mathrm{m}$, leading to large error bars. We observe in the log-log plot of figure $7 \mathrm{a}$ that all the experimental data gather and align well along a straight line of slope $2 / 3$ as predicted by model equation (4.4). This means that the scaling predicted by the model captures the essential of the physical processes with the different physical parameters, $V_{i}, e, b, \phi, R, \rho_{s}$ and $E_{w}$, with scaling laws that differs from the classical Hertz model. However we observe that the experimental data are systematically and significantly below the model prediction that is shown by the continuous line without any fitting parameter. This may come from fluid dissipation which has been neglected in the present modelling.

In figure $7 \mathrm{~b}$, the collision duration $\tau_{c_{0}}$ normalized by the time scale $\left(e R^{2} / \phi\right)^{1 / 3} / c^{*}$ is plotted as a function of the normalized impact velocity $V_{i} / c^{*}$. We also observe here that all the experimental data gather and align well along a straight line of slope $-1 / 3$ as predicted by model equation (4.5). The data for time collision are here above the 
model prediction, in relation to the data for penetration observed to be below the model predictions. This may again be attributed to dissipation.

Let us now look at the importance of gravity in the present experiments. In equation (4.6), the effect of gravity on the maximal penetration can be interpreted as increasing the zero gravity penetration $\delta_{m_{0}}$ by the correction factor $\left(1-3\left(\delta_{s} / \delta_{\text {max }}\right)^{2}\right)^{-1 / 3}>1$. In the present experiments, the ratio $\delta_{s} / \delta_{\text {max }}$ increases from 0.11 to 0.37 with decreasing values of $V_{i} / c^{*}$ so that the correction factor thus varies from 1.02 at high impact velocities up to 1.2 at low impact velocities. Concerning the collision duration, equation (4.7) shows that the effect of gravity is to decrease the values of $\tau_{c_{0}}$, by the correction factor $I_{g} / I_{0}<1$, that decreases in the present experiments from 0.87 to 0.75 with decreasing values of $V_{i} / c^{*}$.

\section{Conclusions}

We have measured precisely, using a high-frequency interferometric technique, the dynamics of collision of a liquid-immersed sphere onto a textured wall comprised of a array of square micro-pillars. With this interferometric technique, micro-rebounds of the sphere down to a few $\mu \mathrm{m}$ can be detected. The results show that the critical Stokes number for bouncing depends on both the aspect ratio and surface density of the pillars, and that its values are smaller than the critical value of 10 reported for a smooth wall. In the bouncing case, the interferometric technique allows us to accurately measure the maximal penetration depth and the duration of the sphere/pillars collision. These measurements have been found in good agreement with the predictions of a collisional model, which is based on the elastic deformation of the pillars and is thus somewhat different from the classical Hertz scalings valid for a smooth wall. The viscous dissipation during the collision has been neglected in a first step in the present modeling, but there is evidence of such a dissipation during the collision. The analysis of the viscous dissipation by the squeezing flow through the network of pillars during the collisional process is ongoing. The interferometric technique that gives access to the dynamics during the collision contact could conclude if unsteady forces, such as added mass and history integral, considered recently by Simeonov (2015) are indeed important to consider for the characterization of immersed collision processes. A precise criterion for bouncing may also be inferred from a detailed knowledge of the texture-modified dynamics of approach.

\section{Acknowledgments}

The authors wish to thank G. Teissier for help with the experimental set-up and F. Restagno for fruitful discussions. T. C. acknowledges financial support of MRES (Ministère de la Recherche et de l'Enseignement Supérieur).

\section{REFERENCES}

Ardekany, A. M \& Rangel, R. H. 2008 Numerical investigation of particle-particle and particle-wall collisions in a viscous fluid J. Fluid Mech. 596, 437-466.

BARNOKY, G. \& DAVIS, R. H. 1988 Elastohydrodynamic collision and rebound of spheres: Experimental verification. Phys. Fluids 31, 1324-1329.

Cassar. C., Nicolas, M. \& Pouliquen, O. 2005 Submarine granular flows down inclined planes Phys. Fluids 17103301.

Chastel, T. 2015 Interactions hydrodynamiques entre une sphère et une paroi texturée : approche, collision et rebond PhD thesis, Université Pierre et Marie Curie.

Chastel, T. \& Mongruel A. 2016 Squeeze flow between a sphere and a textured wall Phys. Fluids 28023301. 
Costa, P., Boersma, B.J., Westerweel, J. \& Breugem, W.-P. 2015 Collision model for fully resolved simulations of flows laden with finite-size particles. Phys. Rev. E 92, 053012.

Courrech du Pont, S., Gondret, P., Perrin, B. \& Rabaud, M. 2003 Granular Avalanches in Fluids. Phys. Rev. Lett. 90, 044301.

Davis, R. H., Serayssol, J.-M. \& Hinch, E. J. 1986 The elastohydrodynamic collision of two spheres. J. Fluid Mech. 163, 479-497.

Falcon, E., Laroche, C. Fauve, S. \& Coste, C. 1998 Behavior of one inelastic ball bouncing repeatedly off the ground. Eur. Phys. J. B 3,, 45-57.

Gondret, P., Hallouin, E., Lance, M. \& Petit, L. 1999 Experiments on the motion of a solid sphere toward a wall: From viscous dissipation to elastohydrodynamic bouncing Phys. Fluids 11, 2803-1999.

Gondret, P., Lance, M. \& Petit, L. 2002 Bouncing motion of spherical particles in fluids. Phys. Fluids 14, 643-652.

Izard, E., Bonometti, T. \& LACAze, L. 2014 Modelling the dynamics of a sphere approaching and bouncing on a wall in a viscous fluid. J. Fluid Mech. 747, 422-446.

Johnson, K.L. 1985 Contact Mechanics., Cambridge University Press, Cambridge.

Joseph, G. G. 1998 Cavitation and the state of stress in a flowing liquid J. Fluid Mech. 366, 367-378.

Joseph, G. G., Zenit, R., Hunt, M. L. \& Rosenwinkel, A. M. 2001 Particle wall collisions in a viscous fluid. J. Fluid Mech. 433, 329-346.

Landau, L.D. \& Lifshitz, E.M. 1970 Theory of Elasticity, Elsevier, New York.

Lecoq, N., Feuillebois, F., Anthore, N., Anthore, R., Bostel, F. \& Petipas, C. 1993 Precise measurement of particle-wall hydrodynamic interactions at low Reynolds number using laser interferometry Phys Fluids A 5, 3-12.

Legendre, D., Zenit. R., Daniel, C. \& Guiraud, P. 2006 A note on the modelling of the bouncing of spherical drops or solid spheres on a wall in viscous fluid Chem. Eng. Sci. 61, 3543-3549.

Lian, G., Adams, M. J., \& Thornton, C. 1996 Elastohydrodynamic collisions of solid spheres. J. Fluid Mech. 311, 141-152.

Marshall, J. S. 2011 Viscous damping force during head-on collision of two spherical particles. Phys. Fluids 23, 013305.

Marston, J. O. ,Yong, W. \& Thoroddsen, S. T. 2010 Direct verification of the lubrication force on a sphere travelling through a viscous film upon approach to a solid wall. J. Fluid Mech. 655, 515-526.

Marston, J. O. ,Yong, W. ,NG, W. K., Tan,R. B. H. \& Thoroddsen, S. T. 2011 Cavitation structures formed during the rebound of a sphere from a wetted surface. Exp Fluids 50, $729-746$.

Mongruel, A., Lamriben, C., Yahiaoui, S. \& Feulllebois, F. 2010 The approach of a sphere to a wall at finite Reynolds number. J. Fluid Mech. 661, 229-238.

Simeonov, J.A. \& Calantoni. J. 2012 Modeling mechanical contact and lubrication in Direct Numerical Simulations of colliding particles. Int. J. Multiphase Flow 46, 38-53.

Simeonov, J.A. 2015 The unsteady hydrodynamic force during the collision of two spheres in a viscous fluid. Acta Mech. 227, 565-580.

YAng, F.-L \& Hunt, M. L.. 2008 A mixed contact model for an immersed collision between two solid surfaces Phil. Trans. R. Soc. A 366, 2205-2218. 